Janusz Mastalski

The Pontifical University of John Paul II in Cracow, Poland

\title{
Genitori dei figli innamorati (tattiche educative)
}

\section{Guidelines for Parents on Raising Enamored Children}

\begin{abstract}
In the contemporary global context where people devalue tradition, the challenge which faces many parents is how they can be meaningfully present in their children's lives. This can be problematic as in the contemporary world children are often searching for their own identity and space which they do not necessarily locate within their own family home. This text explores the question: how can family ties be nurtured to include children's interests and affections? The author provides specific guidelines for raising children so that parents can accompany their children's emotional development.
\end{abstract}

\section{Keywords}

Family, bonds/ties, emotional development.

Il problema della presenza dei genitori nella vita dei loro bambini, nei tempi della globale evasione dalla tradizione, diventa un problema sempre piu inquietante, perche' il figlio o la figlia contemporanei cerca il proprio posto non necessariamente nella casa paterna. Il genitore contemporaneo deve affrontare una sorte di "fuga"dei figli nel mondo delle fascinazioni e avventure dei loro coetanei. Questo mondo e' spesso uno spazio incomprensibile per una persona di un'altra generazione. Proprio in famiglia il bambino dovrebbe sentire uno specifico legame il cui frutto e' la coscienza di essere compreso. Ogni genitore deve ricordare che "il bambino, nascendo, assume il diritto all'appartenenza ottenendo in questo modo il posto in famiglia. Quest'appartenenza crea un 


\section{The Person and the Challenges \\ $94 \quad$ Volume 2(2012) Number 2}

legame fra i genitori e i figli"'. Grazie a questo legame cresce una specifica e intima area degli influssi reciproci che hanno un ruolo molto importante per lo sviluppo e per la formazione delle funzioni sociali della giovane generazione. Fra diversi compiti legati a quest'area c'e' l'adeguato sostegno dei figli nella scoperta di una sottile realta' che e' l'amore.

Prima pero' di formulare un insieme delle concrete tattiche educative riguardanti influsso pedagogico dei genitori sui loro figli, vale la pena di rendersi conto d'alcuni fenomeni che deformano il processo educativo in famiglia, eppure "il primo ambiente, percio', in alcuni aspetti, quello che forma di piu' lo sviluppo del bambino, e' la famiglia che nell'educazione dei figli puo' raggiungere molto ma anche sprecare tanto"2. Quindi sia il padre che la madre, indipendentemente dall'eta' del bambino, dovrebbe ricordare delle seguenti tossiche condizioni della civilizzazione:

- Il genitore contemporaneo sempre piu`spesso trascura i figli di conseguenza ad un tossico stile di vita in cui il consumo, l'inseguimento al denaro e l'esistenza traumogena influiscono sulle interazioni familiari.

- L'uomo contemporaneo molto spesso deve affrontare diversi fattori che furtivamente generano il fenomeno di psicomicidio il quale si manifesta prima di tutto nella disfunzionalita della psiche umana.

- Nei masmedia l'ambiente familiare e' sistematicamente emarginato e cio influisce negativamente sull'aiuto nello sviluppo di tutti i membri della famiglia, eppure "il primo luogo dove inizia il processo educativo di un giovane è la casa paterna"3.

- In numerose famiglie domina un'atmosfera disforica nella quale non c'e' posto per il dialogo, confessioni, neanche per stare insieme più a lungo.

- Il conflitto di generazioni che esiste in famiglia "da sempre" accresce di consequenza di reciproche reticenze e pregiudizi provocati dai lanciati (anche nei masmedia) stili di essere un giovane moderno e globale.

- In molte famiglie si possono incontrare sempre piu numerosi bambini ed adolescenti che non se la cavano con diverse sfide da parte della scuola, dei genitori, dei coetanei. Bisogna pero` ricordare che, nelle situazioni in cui il bambino ha delle difficolta` nello studio, difetti congeniti di sviluppo oppure

${ }^{1}$ J. Jagieła, Relacje w rodzinie a szkoła, Kraków 2007, p. 13.

${ }^{2}$ D. Wall, Twórcze wychowanie w okresie dzieciństwa, Warszawa 1986, p. 3.

${ }^{3}$ Jan Paweł II, Homilia podczas Mszy Świętej, (Łowicz 14.06.1999, nr 2), in: Bóg Jest Miłościa - VII Pielgrzymka Jana Pawła II do ojczyzny, Olsztyn 1999, p. 194. 
qualsiasi qualita psichiche o fisiche per le quali si sente male, cresce la probabilita' d'abusi ${ }^{4}$.

In questo contesto nasce nel giovane uomo una nostalgia sempre piu' profonda di un vero amore, di vicinanza e una durevole amicizia. Dall'alta parte, in quantita spaventose, aumenta il numero di divorzi fra cui ci sono coppie giovani, con una pratica molto breve.

Cresce allora la domanda: dove sta' l'errore? E' uno sbaglio o il periodo di fidanzamento mal approfittato? La risposta sembra molto piu' complicata e sta nell'intero processo dell'educazione del bambino all'amore e tramite l'amore. Vale la pena, percio', dare piu' attenzione al ruolo dei genitori prendendo in considerazione ogni singola tappa dello svilupo umano.

\section{Primi passi nella scoperta dell'amore (periodo d'infanzia)}

Nell'Esortazione di Giovanni Paolo II Familiaris consortio si trova un'indicazione molto concreta, secondo la quale "l'educazione all'amore come dono di sé costituisce anche la premessa indispensabile per i genitori chiamati ad offrire ai figli una chiara e delicata educazione sessuale. Di fronte ad una cultura che «banalizza» in larga parte la sessualità umana, perché la interpreta e la vive in modo riduttivo e impoverito, collegandola unicamente al corpo e al piacere egoistico, il servizio educativo dei genitori deve puntare fermamente su di una cultura sessuale che sia veramente e pienamente personale"s. Indubbiamente, l'educazione all'amore, ma anche al fidanzamento e al matrimonio, e' un'occasione di scoprire non solo la propria sessualita', ma anche un ricco spazio dei sentimenti. Gia', dai primi anni della vita del bambino, i genitori possono in maniera molto sottile aiutare i loro figli a conoscere il mondo dell'amore. Un elemento specialmente importante in questa "spedizione conoscitiva" costituiscono i gesti, che dovrebbero essere coerenti cioe' rappresentare proprio quello che in verita` significano. L'educazione all'autenticita' nei gesti e' il primo e molto concreto passo nell'edificare di un non falso amore. Il bambino che e' stretto al petto ma anche che stringe al petto scopre il valore della necessita` di sicurezza e reciprocita'. Il gesto di stringere al cuore e' l'entrata all'orbita delle interazioni pieni di comprensione reciproca, di un senso di calore e di franchezza. Inoltre, un ruolo molto importante per bambino nel periodo d'infanzia ha il bacio. Questo

${ }^{4}$ R.L. Simons, L.B. Whitbeck, R.D. Conger, W. Chyi-IN, International Transmission of Harsh Parenting, "Developmental Psychology" 27 (1991), p. 159-160.

${ }^{5}$ Familiaris consortio 37. 
gesto unisce in se dimensione di tenerezza con quella di innocenza e purezza. Il bambino che e' baciato, ma anche da' dei baci scopre un mondo dei gesti che manifestano la tenerezza e l'amore che non capisce ancora fino in fondo, pero' si rende conto del suo grande valore.

Un altro aspetto dell'educazione all'amore e' il rinforzamento della volonta', perche' la durabilita' del matrimonio basa non solo sull'amore compreso come sentimento, ma soprattutto come "una scelta". Questa scelta presuppone la fedelta' e la consequenza. Il bambino educato a scegliere conseguentemente rinforza allo stesso tempo la propria forte volonta' e per questo scopre la verita sull'amore che esige dall'uomo il sacrificio e la perseveranza. I genitori che sono gli educatori conseguenti e che esigono la stessa conseguenza dai bambini formano molto importante fondamento della giovane personalita' - capacita' di mantenere equilibrio fra i sentimenti e la ragione. In questo modo viene creata la personalita di un bambino emotivamente intelligente.

Alle regole dell'educazione dei bambini emotivamente intelligenti bisogna inclludere le indicazioni seguenti:

I. "Essere coscienti dei propri sentimenti e di quelli degli altri.

II. Esprimere l'empatia e la comprensione rispetto alle opinioni degli altri.

III. Cavarsela in modo positivo con diversi impulsi emozionali e beavioristici.

IV. Disporsi alla realizzazione dei progetti e degli scopi positivi.

V. Nelle relazioni interpersonali sfruttare delle capacita" sociali positive"6.

I genitori, come e' stato dimostrato, se vogliono eseguire i loro doveri quando i bambini s'innamorano, devono molto prima, tramite l'educazione nell'infanzia, curare il giusto approcio all'amore. L'atteggiamento dei genitori verso i figli nel periodo d'adolescenza deve essere una continuazione dell'educazione nell'infanzia. E' un tempo di prova per tutte e due le parti dalla quale bisogna uscire vincenti.

\section{Prime avventure (periodo d'adolescenza)}

Nel periodo d'adolescenza e' molto importante costruire delle relazioni in cui verra' introdotto un nuovo elemento che e' "la sincerita' nonostante tutto". Gli adolescenti hanno spesso tentazione di tenersi a distanza dai loro genitori, perche' vogliono in questo modo manifestare la propria indipendenza. Esiste

${ }^{6}$ L. Wiatrowska, Próba pedagogicznej terapii w zakresie emocji dysfunkcyjnych uczniów klas niższych szkót podstawowych, in: T. Borowska (ed.), Pedagogika wobec zagrożeń, kryzysów i nadziei, Kraków 2002, p. 160. 
quindi bisogno di una speciale cura da parte dei genitori per creare diverse opportunita per conversazioni sincere e piene di comprensione. Questa tattica educativa e' stata definita da T. Gordon nelle parole seguenti: " Tu ed io stiamo nello stesso legame, nella relazione che ci tengo molto e che vorrei mantenere (...). Voglio cercare di soddisfare onestamente i tuoi bisogni - anche quando hai problemi di soddisfare i propri bisogni e desideri. Se mi permetti di partecipare nei tuoi problemi cerchero' di ascoltare con comprensione e accettazione in tale modo che ti permettera' di trovare la tua soluzione invece di dipenderti dalle mie" nella scoperta dell'amore il quale, se ricambiato, obbliga, perche' questo periodo e' anche il tempo di nascita dei nuovi, sconosciuti sentimenti, altresi delle inquietanti pero' affascinanti reazioni fisiologiche dell'organismo.

I genitori allora come i primi dovrebbero rimanere seri ed eliminare qualsiasi situazione imbarazzante per l'adolescente. Le discussioni sul sesso o su un amore o una affascinazione nascente non possono essere emarginate. Nelle conversazioni intime assolutamente non si puo ' permettere di deridere o disprezzare i problemi riguardanti cosiddetto "stare insieme". Le prime avventure "maschio-femmina" sono molto spesso impacciate e piene di una forte carica emozionale in cui di solito manca la ragione. Proprio per questo c'e' un grande bisogno di ascoltare attentamente e con pazienza ogni comunicato dell'adolescente e di una sottile trasmessa di opinioni che preferiscono atteggiamenti adeguati. Questo è specialmente importante nell'epoca di sempre piu' grandi problemi nella sfera sessuale.

Negli ultimi anni la sessualita' diventa "una zona dei drammatici problemi di

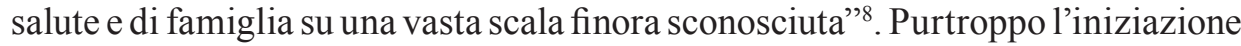
sessuale avviene sempre piu presto. Molto interessanti sono anche gli interventi dei giovani stessi che motivano la propria decisione di avere rapporti sessuali. Secondo J.McDowell si possono elencare le motivazioni seguenti:

1. Il sesso mi fa piacere.

2. Sono sotto la pressione del mio partner.

3. L'avevo gia fatto una volta perche' non farlo ancora?

4. Sono sotto la pressione dei miei coetanei.

5. Non so fino in fondo che cosa' sia l'amore.

6. Sono ribelle all'altra persona.

7. Sono curioso come e :

\footnotetext{
${ }^{7}$ T. Gordon, Wychowanie bez porażek w szkole, Warszawa 1995, p. 284-285.

${ }^{8}$ Sz. Grzelak, Profilaktyka ryzykownych zachowań seksualnych młodzieży, Warszawa 2006, p. 17.
} 
8. E' la mia espressione dell'amore e del bisogno di esere amato?.

A questo punto bisogna ricordare che le differenze nel periodo d'adolescenza si riducono ad un relativo calo dell'importanza di fratelli come fonte dell'intimita' e dell'aiuto. Questo ruolo viene assunto da colleghi, colleghe e amici ${ }^{10}$. In un certo senso anche i genitori diventano per l'adolescente lontani perche' hanno un dovere molto importante di eliminare quegli atteggiamenti che favoriscono dei comportamenti deformanti il modello dell'amore non tossico.

Vale la pena ricordare l'insuperabile ruolo della famiglia negli atteggiamenti prosociali dell'adolescente. Si possono elencare almeno alcuni dei comportamenti dei genitori: fornire chiare regole e principi, approcio emozionale ai figli, attribuire al bambino caratteristiche prosociali, modellare atteggiementi dei figli e una cura empatica ${ }^{11}$. Tramite una disreta vigilanza, il controllo del tempo che i figli passano fuori casa e la creazione di un positivo approcio alla castita', si compone un caratteristico ethos dell'adolescente innamorato che "non e' malato d'amore o a causa d'amore" ma tratta questo stato d'animo come una bellissima avventura di due persone, in cui la prudenza, il rispetto della dignita' altresi ' la capacita' di rinunciare "all'usufruo" dell'altra persona costituiscono i fondamentali principi dell'entrata nelle relazioni intime.

L'adolescente innamorato durante il colloquio con i genitori dovrebbe sentire le non imposte e piene di comprensione parole d'aiuto. Nel caso opposto "le questioni maschio-femmina" si danno alla macchia e diventano un tabu. La madre o il padre si fermeranno sulla fase di sospeti e di un eventuale sogguardo dell'ambiente dei coetanei dei loro figli.

Quanto importante, in questo periodo di nascita dell'amore, e' la testimonianza dei genitori stessi. L'adolescente dovrebbe di nuovo (come uomo giovane) rileggere il messaggio verbale e non verbale dei propri innamorati genitori. Nell'atteggiamento del padre e della madre dovrebbe scoprire l'essenza dell'amore. Non e' soltanto un'affascinazione che da' la gioia e la felicita'. L'amore e' anche la responsabilita', la capacita' di rinunciare, lo spirito di sacrificio, la fedelta' altresi ' la volonta' di entrare sul piano di dialogo in cui c'e' posto per compromessi e per necessita' di dare se stessi l'uno all'altro.

La famiglia e' un insostituibile "poligono" dei comportamenti che approfondiscono l'amore. I genitori che si rivolgono l'uno all'altro con il rispetto

\footnotetext{
${ }^{9}$ J. McDowell, Nie! Pomóż swojemu dziecku pokonać presję seksualna, Lublin 1993, p. 33-34.

${ }^{10}$ D. Burmester, W. Furman, Perceptions of Sibiling Relationships During Middle Childhood and Adolescence, "Child Development" 61 (1990), p. 1387-1398.

${ }^{11}$ J.L. Robinson, C. Zahn-Waxler, R. Emde, Patterns of Development in Early Empathic Behavior: Environmental and Child Contributional Influences, "Social Development" 3 (1994), p. 125-145.
} 
e la bonta' fanno vedere ai figli adolescenti aspetti pratici dell'amore. Percio l'adolescente ha l'occasione di prendere l'esempio dai suoi genitori. Molto spesso il modo di parlare fra la madre e il padre viene trasferito sulla relazione: l'adolescente - la sua simpatia. I genitori, con il loro giusto comportamento, devono opporsi al modello della relazione di due persone lanciata anche nei masmedia: velocemente - piacevolmente - senza impegno. Nasce quindi una nuova categoria del pseudo - amore, che non ha niente in comune con il dono di se stessi, ne' con il rispetto ne' con la soggettivita'.

Avviene pero' il momento quando i genitori vedono che il loro figlio o la loro figlia trattano sempre piu’ seriamente la relazione affettuosa. E' una straordinaria sfida per i genitori, perche' devono affrontare il fatto che il loro bambino cresce e che a poco a poco ma sistematicamente "andra' via" da casa. Ci vuole a questo punto una grande maturita` e lungimiranza.

\section{La fatica dell'edificare una nuova relazione (periodo di "stare insieme")}

Nei masmedia si parla piu’ spesso della cosiddetta educazione comercializzata, che e' un tipo di educazione concentrata "sull'effetto inteso non come desiderato dalla societa', ma come spettacolarita' dello scopo e del succeso. Il riconoscimento dell'efficacia come l'attributo principale dell'educazione fa che il processo di educazione deve essere redditizio e non puo portare perdite. In questo significato l'educazione e' un investimento, che rende profitti e rimunera. L'educazione comercializzata dovrebbe quindi essere prevedibile, vuol dire che bisogna minimalizare, anzi eliminare tutti i casuali e non premeditati influssi considerati dalla socializzazione" ${ }^{\prime 2}$. Molto spesso i genitori guardano il loro adolescente o adulto figlio/-a attraverso il prisma delle possibilita di raggiungere il successo. Sulla strada che ci porta il/la partner del loro bambino puo` disturbare oppure aiutare. Allora nel periodo di "stare insieme con qualcuno" il ruolo dei genitori e' indispensabile. Prima di tutto non possono commettere errori che si riflettano nella relazione del loro bambino.

Il primo errore cardinale e' il cedere alla sindrome della prima impressione. Esiste la tentazione di valutare l'uomo a prima vista. Il primo incontro puo' provocare cosiddetto "effetto hallo" per cui si quarda l'uomo tramite la prima cantonata o una brutta figura ecc. Questa prima impressione porta a schedare un eventuale prossimo genero o prossima nuora. Di conseguenza viene curata

${ }^{12}$ U. Kusio, Czym jest wychowanie skomercjonalizowane, „Małżeństwo i Rodzina” 1-2 (2005), p. 24. 
un'avversione al partner di figlio/a che si fa vedere soprattutto nel comportamento e nelle opinioni su di lui/lei. Il figlio innamorato sente piu' forte discomforto perche' vede la mancata accettazione da parte dei genitori del suo partner. Di conseguenza questo "stare insieme" diventa il vivere l'amore di nascosto.

Un altro atteggiamento tossico dei genitori e' la paura di perdere il figlio adulto. E' una gelosia di concorrente. Il figlio o la figlia innamorati passano piu tempo fuori casa con la persona amata e i genitori vengono trascurati. Avvengono allora molti rimproveri o infondate avvetenze che prima o poi inconsciamente diventano una svalutazione del partner negli occhi del figlio/a.

Il terzo errore dei genitori e' la tendenza ad essere insistenti e troppo curiosi. La madre o il parde che costringono i figli innamorati a confessare particolari degli incontri "spogliano" la relazione giovanile da un'indispensabile aria di misteriosita' e intimita'. A questo atteggiamento molto spesso e' legata la tendenza di demonizzare la relazione mostrando ai figli soltanto vari pericoli. Trattare il partner del figlio/a come un potenziale "imbroglione/a" che non ha buone intenzioni e' un giudizio parziale e molto spesso ingiusto.

In questo modo nasce il conflitto che e' pero' un naturale processo il quale avviene nell'ambito di tutte le relazioni importanti, fa parte della loro natura ed e' soggeto ad un costruttivo regolamento tramite la comunicazione ${ }^{13}$. Tale comunicazione deve essere di tipo appoggiante-informativo. I genitori dovrebbero piuttosto diventare consultanti e non soltanto quelli che decidono.

Gli innamorati adolescenti in un colloquio con i loro genitori devono sentire certe verita primarie riguardanti la creazione di una stabile e durevole relazione. Una di queste regole e' il legame fra l'amore e la fede. Il giovane uomo deve scoprire che "l'amore non solo tratta l'altro bene, ma anche lo rende buono. Ispira la bonta'scoperta dalla fede guardando la realta' da un'altra parte. L'amore trasforma la realta', la rende buona, forma in essa la bonta'. La fede permette di vedere in un'altra ottica, l'amore trasforma"'14.

I genitori dovrebbero aiutare i loro figli di seguire il cammino di vocazione al matrimonio il che vuol dire: "imparare l'amore degi sposi di giorno in giorno, di anno in anno: la carita'secondo l'anima e corpo, la carita' che e' paziente e benigna, che non cerca il suo interesse, non tiene conto del male ricevuto, la carita che sa compiacersi della verita', la carita' che tutto sopporta"15. E' molto importante una personale testimonianza dei genitori che sanno dialogare tra di loro basando sulla comprensione reciproca e sul perdono.

\footnotetext{
${ }^{13}$ J.L. Hocker, W.W. Wilmat, Interpersonal Conflict, Dubuque IA 1985, p. 6.

${ }^{14}$ A. Grün, Kiedy wstuchuje się w Boga, Kraków 2002, p. 66-67.

${ }^{15}$ Jan Paweł II, List Apostolski Parati semper, Kraków 1985, Nr 10.
} 
Bisogna sapere che la disintegrazione emozionale di famiglia ha un influsso negativo sullo sviluppo psichico del bambino tanto in famiglia con ambedue i genitori quanto nella famiglia dove ha avuto luogo il divorzio ${ }^{16}$. E' quindi indispensabile creare un legame emozionale in famiglia, perche` essa garantisce in futuro buone relazioni con i suoceri e una speciale cura per le interazioni familiari piene di amore e calore. Un forte legame familiare incoraggia all'autofromazione seguendo il modello di marito rappresentato dal padre o quello di moglie visto nell'atteggiamento della madre.

La dimensione del legame familiare puo' perfettamente far vedere un e-mail di un giovane uomo che ha aprezzato dopo anni la sua casa paterna: "Ormai sono un uomo adulto, alla soglia del matrimonio. Ci sono voluti 24 anni per farmi capire quanto importane e` stata per me la famiglia. Non ho mai apprezzato veramente i miei genitori, soprattutto mia madre. Solo adesso, quando si trova in ospedale comincio a rendermi conto che se la perdessi sarebbe per me un colpo durissimo. Ricordo dall'infanzia i suoi occhi pieni di lacrime quando ho combinato un'altra volta un guaio. Ricordo quando entrava nella mia stanza per controllare se tutto andava bene. Ricordo il suo sguardo intelligente quando le volevo dimostrare che non aveva ragione. Oggi guardo anche in altra maniera mio padre. Era severo, qualche volta inaccessibile, dall'altra parte pero` dava il senso di sicurezza, di qualche stabilita'. Oggi ha piu’ di cinquanta anni, pero' sempre si puo' seguire il suo esempio. Solo che quando avevo 12 o 15 anni lui mi sembrava "un apparato d'opressione". Dopo anni apprezzo pienemente la sua severita', ma anche l'amore. Amo oggi i miei genitori in modo piu' maturo. Peccato che solo cosi' tardi ho scoperto questo tesoro, il mio legame d'amore con loro".

Questo esempio di un giovane ragazzo fa vedere che l'apprezzamento dei genitori avviene qualche volta piu' tradi, percio' bisogna in modo delicato e discreto aiutare bambino nel periodo d'adolescenza e prepararlo alla decisione di matrimonio. Questo compito finisce il giorno di fidanzamento, perche’ cambia il suo ambito.

\section{Tempo di preparazione diretta (periodo di fidanzamento)}

Nel 1996 Giovanni Paolo II ha detto ai giovani: "Vi chiedo di diventare «profeti della vita». Siatelo con le parole e con i gesti, ribellandovi alla civiltà dell' egoismo che spesso considera la persona umana uno strumento anziché un

${ }^{16}$ N. Kalter, Long-Term Effects of Divorce on Children. A Developmental Vulnerability Model, „American Journal of Orthopsychiatry” 57 (1987), p. 587-597. 


\section{The Person and the Challenges \\ 102

fine"17. Il periodo di fidanzamento e' un tempo in cui si allarga la prospettiva della lotta contro il proprio egoismo. E' anche il tempo per prepararsi ad un definitivo cambiamento del pensiero. La categoria io deve essere sostituita da quella del noi. Percio' Benedetto XVI ha sottolineato: "Voi, giovani fidanzati, vivete il fidanzamento nell'amore vero, che comporta sempre il reciproco rispetto, casto e responsabile"18. In questa tappa i genitori devono adempiere un ruolo molto importante per aiutare i figli a realizzare le soppracitate parole del papa. Ecco alcuni principi che delineano il servizio dei genitori verso i fidanzati.

1. Principio di consiglio non invadente. Il periodo di fidanzamento e' un tempo molto importante per i fidanzati e allo stesso momento molto bello. E' il tempo di molti progetti e della loro prossima realizzazione. In tale fervore di diverse attivita' preparative esiste il pericolo di troppa fretta, poca ragionevolezza, di prendere decisioni in modo inconsiderato. Percio' i genitori dovrebbero essere il baluardo della pace e una fonte di suggerimenti.

2. Principio di creazione di legami con il nuovo, futuro membro di famiglia. Molti fidanzati hanno paura della mancata accettazione da parte dei suoceri. I genitori, tramite l'atteggiamento di sincerita' e autenticita possono diminuire queste ansie. I lavori fatti insieme (preparazione di pasti, lavaggio di macchina, pulizie ecc.), il tempo libero passato insieme e discussioni su diversi temi assuefanno genero o nuora che si sentono piu accettati e diventano in qualche senso compadroni. Allo stesso momento il figlio o la figlia si sentono bene perche' il suo fidanzato/a riesce a capirsi con i genitori.

3. Principio di eliminare troppa ingerenza. Nel periodo di fidanzamento si cristalizza il senso di autonomia, percio` per molti fidanzati nasce un grande bisogno di decidere in molte questioni. La saggezza di genitori esige da loro stessi la maturazione alla coscienza che il loro bambino ha diritto di avere la propria oponione e persisterci. C'e' pero' una tentazione di immettersi con pretesto di voler aiutare, di avere piu' grande esperienza o di vedere un'incapacita' dei fidanzati. I genitori che impostano la loro volonta' ai fidanzati, che non prendono in considerazione l'opinione dei propri figli (p.es. riguardante la festa di nozze) diventano un appoggio tossico.

4. Principio del mostrare l'amore magnanimo. E' un amore volizionale perche' la capacita' spirituale della volonta' permette all'uomo volgersi verso la bonta' universale e quella del prossimo. Dall'altra parte "amore emozionale e' per

${ }^{17}$ Jan Paweł II, Orędzie na XI Światowy Dzień Młodzieży, „L’Osservatore Romano” (wyd. pol.) 2 (1996).

${ }^{18}$ Benedykt XVI, Przemówienie w czasie liturgii pokutnej dla młodzieży diecezji rzymskiej (29.03.2007), „L'Osservatore Romano” 5 (2007). 
la sua natura indirizzato verso se stessi; non e' in grado di essere di per se' magnanimo e altruistico (...). E' l'amore magnanimo che rende l'uomo capace di superare se stesso e di pensare prima di tutto al bene del prossimo". ${ }^{19}$ Per amare in questo modo ci vuole un riferimento che e' Cristo. Si tratta di conseguenza di un'atmosfera creata dai genitori dell'amore che dona, che offre, che e' una concreta realizzazione del comandamento d'amore di Cristo. In tale amore non c'e' posto per manipolazione che provoca spesso varie disfunzioni di personalita' fra cui anche la diminuzione dell'autovalutazione ${ }^{20}$. I genitori devono allora smascherare ogni sintomo di un trattamento oggettivo oppure di una manipolazione di emozioni (far compassione, persistere nella pripria opinione e ricattare ecc.).

5. Principio d'affermazione di tenerezza. Nel periodo di fidanzamento c'e' una gande tentazione di oltrepassare ogni principio ed ogni confine legati alla visione cristiana del fidanzamento. Nasce una tentazione di consumare la relazione perche' tanto fra poco sara` matrimonio. La passione rinforzata dal desiderio da' molte occasioni per entrare sulla strada di una permanente lussuria. I genitori, tramite il loro atteggiamento, possono indicare come dimostrarsi l'amore l'uno all'altro tramite la tenerezza che e' l'inverso del desiderio. La tenerezza esprime la preoccupazione per l'altro e allo stesso tempo e' la sensazione e il dono con superiorita' dell'elemento di donare. La tenerezza esprime anche l'affascino per l'altro, l'accettazione dei valori che ha e che rappresenta e in questo modo aumenta la valutazione dell'uomo ${ }^{21}$.

6. Principio dell'accompagnare in preghiera. L'amore sollecita l'uomo ad una piu profonda conoscienza della persona amata. L'amore per Dio in un certo senso "costringe" l'uomo ad un costante conoscienza e approfondimento del Creatore. Questa verita` e' stata rappresentata in maniera molto chiara da C. Caretto nel suo libro "L'amore che sboccia nel deserto": "La preghiera che e' l'amore nell'agire, la dinamica della speranza e il discorso d'amore risulta insostituibile per un reale raggiungimento della vita eterna, cioe` per la vera conoscienza di Dio. Chi non prega non puo' conoscere la profondita' della vita di Dio, non puo` conoscere affatto Dio che e' l'amore. Puo` conoscere Dio soltanto esternamente, come qualche simbolo, qualche idea, filosofia o scienza, come qualche numero segreto, spazio o qualche eternita" 22 . La preghiera non e’ quindi altro che l'immergersi nella vita di Dio. La preghiera dei genitori

\footnotetext{
${ }^{19}$ C. Baars, Psychologia miłości i seksualności, Poznań 2002, p. 30.

${ }^{20}$ A. Martos, No puedo mas. Las mil caras del maltrato psicologico, Castellano 2003, p. 21-22.

${ }^{21}$ T. Jakubowski, Dojrzałość do matżeństwa - wybór wspótmatżonka, „Katecheta” 3 (2000).

${ }^{22}$ C. Caretto, Tajemnice pustyni, Kraków 2002, p. 48.
} 
per i figli fidanzati e' in un certo senso l'atto di entrare nella loro vita. E' una forma di un discreto accompagnamento nella maturazione dell'amore dei loro figli. La comunione per loro, il rosario recitato ogni giorno e l'esempio della preghiera in famiglia creano forti risorse per appoggiare la famiglia.

7. Principio d'integrazione. I genitori nel periodo di fidanzamento dei loro figli dovrebbero essere un fattore integrativo per tutti i doveri, per tutte le scelte e i progetti dei fidanzati. Hanno dunque il ruolo di unire, non dividere. Un saggio suggerimento, la pazienza e il rispetto per la zona intima dei figli adulti e delle loro persone amate sono garanzia di un giusto appoggio dell'amore che unisce i fidanzati. I genitori devono garantire il senso di sicurezza insieme al senso di accettazione. Bisogna ricordare che "un carattere specifico dell'ambiente familiare e' fra l'altro il fatto che le sue funzioni costituiscono un'unita' organica, composta e omogenea, che nella vita quotidiana non possono essere realizzate separatamente. Percio qualsiasi disfunzione che disturba realizzazione di una delle funzioni principali provoca piu’ breve o piu’ lunga incapacita di famiglia al suo generale funzionamento"23. Bisogna quindi cercare di integrare l'ambiente familiare, perche' i fidanzati abbiano la fonte dove ritornare per ottenere concrete indicazioni, conforto e appoggio.

$\mathrm{Da}$ qualche anno ricerche psicologiche svelano molto spaventosi tratti degli orientamenti morali di giovani che piu spesso vengono caratterizzati da: "superficiale orizzonte di vedere il mondo, poverta' di contenuo dello spazio e del mondo in cui vivono le unita', poca resistenza alla manipolazione e influssi non etici, suscettibilita' alla violenza, facilita di sottomettersi alle tentazioni di un facile possesso, pretendere - richiesta di adempire alte esigenze consumistiche senza nessun sforzo personale, passivita e apatia, mancanza di identificazione con i valori sociali, esclusione dall'ambiente pubblico, superficiale e frequentivo approcio alla religione, conformismo e disturbi d'identita" 24 . Tutto il sopraelencato "bagaglio di carico" i giovani portano alla zona d'amore e alla relazione basata sull'amore. I genitori dovrebbero rendersi conto di varie disfunzioni di civilizzazione e gia' da primi anni fare una specifica "detossicazione" dei loro figli. Si tratta quindi di creare, tramite lo stile di vita familiare, un modello di un vero amore che dona, per cui vale la pena sacrificare la propria vita, il tempo, $\mathrm{i}$ talenti. I bambini che crescono con il senso dell'amore che li circonda scoprono da soli il valore d'amore che non e' soltano un'emozione, ma che e' soprattutto un conseguente incontro e qualcosa che dura per sempre.

${ }^{23}$ S. Kawula, Studia z pedagogiki społecznej, Olsztyn 1996, p. 149.

${ }^{24}$ Z. Kwieciński, Pedagogika i edukacja wobec wyzwania kryzysu i gwałtownej zmiany społecznej, in: Z. Kwieciński, L. Witkowski (ed.), Ku pedagogii pogranicza, Toruń 1990, p. 7. 
Alcuni anni fa D.von Hildebrand ha scritto: "Diciamo cosi' quando una persona e` premurosa, quando dimostra benevolenza verso gli altri, quando e` giusta, quando e' pronta a sacrificarsi per gli altri, quando e' compassionevole e magnanima, quando perdona danni subiti ecc. Tutte queste virtu' sono semplicemente speciali forme dell'amore. L'amore e' un flusso della bonta', la bonta' e' un soffio, un alito dell'amore". ${ }^{25}$ I genitori dei figli innamorati dovrebbero diventare la fonte di questo flusso di bonta', che permette di far sentire agli altri di essere amati e che allo stesso tempo suscita il desiderio di amare con l'amore che dona. Da molte ricerche scientifiche risulta che "il livello di simpatia, di generosita', d'amore che il bambino dimostra verso agli altri in gran parte dipende da quanto amore gli e' stato dato nell'infanzia. Gli effetti dell'educazione dei bambini non dipendono dai metodi speciali, ma dal tipo di relazione dei bambini con i genitori" ${ }^{26}$. Amando i propri bambini i genitori creano in loro un progetto d'amore che "per essere pieno, saggio e umano deve unire le emozioni con l'intelletto. Percio' ogni genitore deve conoscere parole come: rinuncia, sforzo di genitore o sacrificio. Dovrebbe anche ricordare che nella vita familiare e' necessaria un'atmosfera che da' ad ogni membro un senso di sicurezza, d'intimita' e dei legami emozionali, un senso che nessuno e' umiliato, impercetibile oppure poco apprezzato" 27.

Bisogna ricordare che se l'essenza d'amore fossero soltanto le emozioni, l'amore sarebbe volubile come le amozioni ${ }^{28}$. La stabilita' dell'amore dei genitori e la loro stabilita' emozionale garantisce il giusto atteggiamento dei genitori verso i loro innamorati figli. E' difficile opporsi all'opinione che in famiglia i bambini vengano educati prima di tutto dalla personalita dei genitori tramite le sue doti positive, tramite i giusti comportamenti sociali, morali, culturali e religiosi, pero' il bambino deve vedere tutto quello, vedere con i pripri occhi diversi tipi di comportamento dei genitori e grazie ad essi formare i propri atteggiamenti" ${ }^{29}$.

25 D. von Hildebrand, Fundamentalne postawy moralne, in: D. Von Hildebrand, J.A. Kłoczowski, J. Paściak, J. Tischner (ed.), Wobec wartości, Poznań 1982, p. 44.

${ }^{26}$ M. Braun-Gałkowska, Psychologia domowa, Olsztyn 1990, p. 66.

${ }_{27}$ J. Maciaszkowa, O wspótżyciu w rodzinie, Warszawa 1980, p. 154.

${ }^{28}$ A. Kępski, Miłość a uczucia, Warszawa 1999, p. 78.

${ }^{29}$ M. Dziewiecki, Pedagogika miłości dla wychowawców i wychowanków, Częstochowa 2001, p. 78. 


\section{Bibliography}

Baars C., Psychologia miłości i seksualności, Poznań 2002.

Benedykt XVI, Przemówienie w czasie liturgii pokutnej dla młodzieży diecezji rzymskiej (29 III 2007), „L'Osservatore Romano” 5 (2007).

Braun-Gałkowska M., Psychologia domowa, Olsztyn 1990.

Burmester D., Furman W., Perceptions of Sibiling Relationships during Middle Childhood and Adolescence, "Child Development" 61 (1990), p. 1387-1398.

Caretto C., Tajemnice pustyni, Kraków 2002.

Dziewiecki M., Pedagogika miłości dla wychowawców i wychowanków, Częstochowa 2001.

Gordon T., Wychowanie bez porażek w szkole, Warszawa 1995.

Grün A., Kiedy wstuchuje się w Boga, Kraków 2002.

Grzelak Sz., Profilaktyka ryzykownych zachowań seksualnych młodzieży, Warszawa 2006.

Hildebrand D. von, Fundamentalne postawy moralne, in: D. Von Hildebrand, J.A. Kłoczowski, J. Paściak, J. Tischner (ed.), Wobec wartości, Poznań 1982, p.7-50.

Hocker J.L., Wilmat W.W., Interpersonal Conflict, Dubuque IA 1985.

Jagieła J., Relacje w rodzinie a szkoła, Kraków 2007.

Jakubowski T., Dojrzałość do matzeństwa - wybór wspótmatżonka, „Katecheta” 3 (2000), p. 20-28.

Jan Paweł II, Orędzie na XI Światowy Dzień Młodzieży, „L’Osservatore Romano” (wyd. pol.) 2 (1996).

Jan Paweł II, Familiaris consortio, Kraków 1981.

Jan Paweł II, Homilia podczas Mszy Świętej, (Łowicz 14 VI 1999, nr 2), in: Bóg Jest Miłościa - VII Pielgrzymka Jana Pawła II do ojczyzny, Olsztyn 1999.

Jan Paweł II, List Apostolski Parati semper, Kraków 1985.

Kalter N., Long-Term Effects of Divorce on Children. A Developmental Vulnerability Model, „American Journal of Orthopsychiatry” 57 (1987), p. 587-597.

Kawula S., Studia z pedagogiki społecznej, Olsztyn 1996.

Kępski A., Miłość a uczucia, Warszawa 1999.

Kusio U., Czym jest wychowanie skomercjonalizowane, „Małżeństwo i Rodzina” 1-2 (2005), p. 21-32.

Kwieciński Z., Pedagogika i edukacja wobec wyzwania kryzysu i gwałtownej zmiany społecznej, in: Z. Kwieciński, L. Witkowski (ed.), Ku pedagogii pogranicza, Toruń 1990.

Maciaszkowa J., O współżyciu w rodzinie, Warszawa 1980.

Martos A., No puedo mas. Las mil caras del maltrato psicologico, Castellano 2003.

McDowell J., Nie! Pomóż swojemu dziecku pokonać presję seksualna, Lublin 1993. 
Robinson J.L., Zahn-Waxler C., Emde R., Patterns of Development in Early Empathic Behavior: Environmental and Child Contributional Influences, "Social Development" 3 (1994), p. 125-145.

Simons R.L., Whitbeck L.B., Conger R.D., Chyi-IN W., International Transmission of Harsh Parenting, "Developmental Psychology” 27 (1991), p. 159-160.

Wall D., Twórcze wychowanie w okresie dzieciństwa, Warszawa 1986.

Wiatrowska L., Próba pedagogicznej terapii w zakresie emocji dysfunkcyjnych uczniów klas niższych szkół podstawowych, in: T. Borowska (ed.), Pedagogika wobec zagrożeń, kryzysów i nadziei, Kraków 2002. 
